\title{
AUSPICE: Automatic Safety Property Verification for Unmodified Executables
}

\author{
Jiaqi Tan, Hui Jun Tay, Rajeev Gandhi, and Priya Narasimhan \\ Department of Electrical \& Computer Engineering, Carnegie Mellon University \\ tanjiaqi@cmu.edu, htay@andrew. cmu.edu, rgandhi@ece.cmu.edu, priya@cs. cmu .edu
}

\begin{abstract}
Verification of machine-code programs using program logic has focused on functional correctness, and proofs have required manuallyprovided program specifications. Fortunately, the verification of shallow safety properties such as memory isolation and control-flow safety can be easier to automate, but past techniques for automatically verifying machine-code safety have required post-compilation transformations, which can change program behavior. In this work, we automatically verify safety properties for unmodified machine-code programs without requiring user-supplied specifications. Our novel logic framework, AUSPICE, for automatic safety property verification for unmodified executables, extends an existing trustworthy Hoare logic for local reasoning, and provides a novel proof tactic for selective composition. We demonstrate our automated proof technique on synthetic and realistic programs. Our verification completes in 6 hours for a realistic 533-instruction string search algorithm, demonstrating the feasibility of our approach.
\end{abstract}

\section{Introduction}

Interactive theorem proving using logic is a promising technique for reasoning about executable (i.e., machine-code) programs, as it provides a succinct specification of the program. However, formally reasoning about machine-code is challenging as accounting for low-level details and writing proofs interactively can be tedious. Logics have been developed to formally reason about the lowlevel state (e.g., registers, main memory) in machine-code programs: Myreen et al. developed a Hoare logic for realistically modeled machine-code [16]. These logics are designed to verify the correctness of programs, and hence must capture the complete execution state of the program, which requires manually supplied specifications e.g., loop invariants, and function pre-/post-conditions. Hence, techniques for reasoning about program correctness ease the job of the proof author [17], but do not fully automate proof generation. Fortunately, verifying shallow safety properties can be easier, as we are only concerned with the parts of program state which affect our desired safety properties. Thus, there are more opportunities for proof automation. Zhao et al. [27] proposed a program logic for automatically verifying safety properties in executables, but programs must be compiled with a modified compiler and safety checks must be added postcompilation [27], thus developers cannot observe how the safety checks added to their programs may change them. 
In this paper, we present a novel logic framework, AUSPICE, for automatically verifying safety properties for unmodified machine-code programs: programs generated by an unmodified compiler, without any post-compilation transformations (e.g., binary rewriting). Thus, any safety checks must be added as source-code statements. This enables developers to gain assurance of their program's behavior and safety from observing the added safety checks. Our contributions are: (i) a novel logic framework, AUSPICE, for automatically verifying safety properties in unmodified machine-code programs, (ii) a logic, $\mathcal{L}_{\mathcal{L R}}$, which enables local reasoning to ensure that safety properties are asserted and checked for every instruction in a machine-code program, (iii) a proof tactic for selective composition which enables the automatic verification of safety properties without manual inputs, and (iv) an empirical evaluation of AUSPICE on verifying real-world machine-code. To the best of our knowledge, AUSPICE is the first logic framework which enables the fully automated proving of safety properties for unmodified ARM machine-code programs, avoiding the post-compilation transformations required by ARMor [27]. We currently target ARM machine-code programs, although our technique can be applied to other architectures.

Intuition. Our safety property verification uses Hoare logic to reason about machine-code. Hoare logic was designed to reason about program correctness, hence, typical Hoare logic proofs must reason about the "global" effects of programs, i.e., capture all possible values of program state. Our first intuition is that our safety properties at each instruction are affected only by the program state immediately before the instruction runs. This enables us to consider only a subset of program state and perform "local" reasoning (§3), and avoid requiring manually supplied specifications. Second, previous efforts to automate safety property verification [27] relied on binary rewriting to insert safety checks. In unmodified machine-code, safety checks must be implemented entirely in sourcecode. Our second intuition is that, when verifying safety properties for unmodified machine-code, safety checks inserted in a program's source-code can span a larger part of a program than our "local" scope of reasoning described above. Hence, we develop a novel proof tactic, selective composition (§4), which uses the Hoare logic Compose rule (§2.4) to help us reason about safety properties using additional contextual information not available in purely local reasoning.

\subsection{Problem Statement}

Goals. The main objective of our logic framework is to automatically prove safety properties for machine-code programs which have been compiled using an unmodified compiler, with no post-compilation modifications (e.g., binary rewriting). The goals of our logic framework are: (i) to use an independently developed, trustworthy logic so that our approach is trustworthy; (ii) to fully automate our proof by not requiring manual inputs from the user, and (iii) to formalize the notion of safety for the execution of a machine-code program.

Non-Goals. We do not intend to prove the correctness of machine-code programs, and we are not concerned with the security and privacy of applications implemented by the machine-code. 
Scope. We choose to verify safety properties for the machine-code of programs rather than their source-code so that (i) we do not need to trust the compiler used, thus minimizing our Trusted Computing Base (TCB), and (ii) our verification does not need access to the source-code of the program. We require no modifications to the compiler used to generate the executables which we verify. Our logic framework currently targets ARM machine-code programs, although our technique can be applied to machine-code for other architectures by (i) parameterizing the Hoare logic [16] with a different instruction semantics, and (ii) defining execution safety for the target architecture. We verify safety properties for user programs running on a commodity operating system (currently Linux). Assumptions. Our logic framework uses the trustworthy formalization of the ARM Instruction Set Architecture (ISA) developed by Myreen et al. [15] at Cambridge University (the "Cambridge ARM model"). Thus, our verification inherits the assumptions and limitations of this model. We assume that the behavior of the program being verified is not affected by exceptions, interrupts, and page table operations, as these are not modeled in the model. We are also unable to verify safety properties in the presence of system calls, as the model does not capture the effects of specific system calls on user programs. We are also unable to verify programs with concurrent behavior, nor unstructured controlflow jumps (e.g., longjmp and switch statements). We assume that the compiler and program obey the ARM-THUMB Procedure Call Standard (ATPCS) [1], which specifies the behavior for function calls/returns, and that the OS correctly isolates concurrently executing user programs. We also assume that the target program being verified was compiled with a well-known, unmodified compiler with well-known function prologues and epilogues, and that the machine-code contains function boundaries. We also require programs to be statically compiled so that all code to be executed is present, and that programs are not recursive.

\section{Background}

\subsection{ARM Architecture}

First, we review aspects of the ARM architecture pertinent to defining execution safety for ARM machine-code programs. ARM is a RISC, load/store architecture, and data instructions operate only on register contents but not memory [2]. There are 6 processor modes, and we focus on the user mode, which an operating system (OS) runs applications in. The remaining modes handle various types of exceptions, including system calls. Each ARM processor mode has a different set of visible registers, and we focus on only the registers visible in user mode: registers $r 0$ through $r 15$, and the status register (CPSR). We also consider the ATPCS [1], which specifies conventions for procedure calls and returns. By the convention in the ATPCS, registers $r 13, r 14, r 15$ store the stack pointer (SP), link register (LR) for return addresses and program counter (PC). We highlight these registers for their impact on control-flow safety. The state of an ARM processor comprises the registers r0 to r15, the processor status register CPSR, and the processor's main memory (modeled as an array of $2^{32}$ byte-addressed bytes). 


\subsection{Safety properties for ARM machine-code programs}

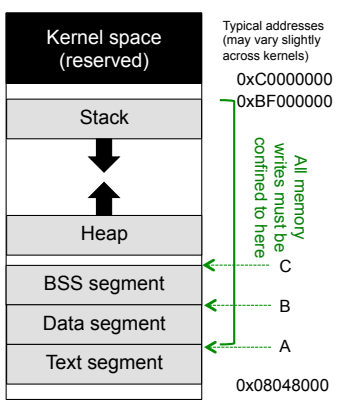

(a) Stack-based Memory safety: Linux Process Memory Layout

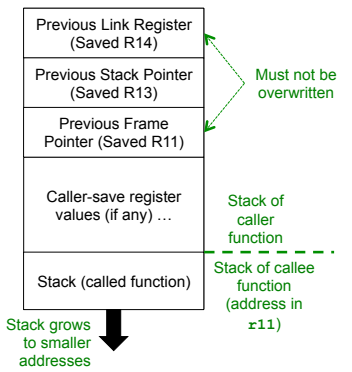

(b) Stack-based Controlflow safety: Function Activation Record

Fig. 1. Safety properties
Next, we discuss the safety properties we wish to prove for ARM machine-code programs. At a highlevel, we wish to prove that the execution of a machine-code program is isolated from any harmful effects of potentially malicious user input. Specifically, we wish to prove that the control-flow of a machine-code program cannot be hijacked and changed at runtime due to user input, and that new behaviors cannot be introduced through code injection or modification. We do this by proving that (i) machine-code loaded to memory cannot be overwritten; that (ii) function-return addresses saved to the program's stack cannot be changed; and that (iii) only machine-code initially loaded to memory is executed. Concretely, at the machine-code level, we prove two safety policies, memory isolation, and control-flow isolation, which together provide the machine-code safety properties sufficient to show that our desired high-level safety property holds. This is similar to Control-Flow Integrity [3], except that we disallow the use of arbitrary function pointers on a program's heap. We instantiate these safety properties in the context of user programs running in an OS (currently Linux), as our goal is to provide isolation for user programs running in an OS.

Memory Isolation. The goal of our memory safety policy, memory isolation, is to prevent a program from modifying its own instructions to prevent the introduction of new behaviors through self-modification. In a multiprogramming OS such as Linux, each user program runs as a separate process with its own virtual memory with a common layout. In processors with 32-bits of addressable memory, each process has a 4 GB memory space, with the upper 1GB reserved for the OS. Figure 1(a) illustrates this layout. Our memory safety policy requires that all memory writes be restricted to the area between the start of the process's stack space (marked 0xBF000000) and the start of the text segment of the code. Control-flow Isolation. The goal of our control-flow safety policy, controlflow isolation, is to ensure that there are no unexpected control-flow transfers, that only instructions in the text section of the program are executed. We also require that there can be no control-flow hijacks via modified function-return addresses. Our memory safety policy partially ensures control-flow isolation by preventing the modification of the text section. Our control-flow isolation is also enforced by protecting the return addresses for function calls saved on the program stack. First, we consider the ATPCS [1] convention. Registers r11 and r13 store the frame pointer (stack base address) and stack pointer (stack top 
address) respectively, while $\mathrm{r} 14$ stores the return address of the current function. When a function call is made, the caller function first saves its current values of r11, r13, and r14 on the stack, before loading the return address to r14. Also, the ATPCS specifies that the stack grows downwards to lower addresses. Thus, to prevent control-flow hijacks, we must ensure that all memory writes are to addresses smaller than the current function's frame pointer (r11) (Figure 1(b)).

\subsection{Hoare logic for ARM machine-code programs}

We use the HOL4 theorem prover [20], and the Hoare logic [16] for ARM machine-code programs [15] developed at Cambridge, to prove safety theorems. The Cambridge ARM model has been extensively tested and validated [9], providing us with a strong, trustworthy foundation for our logic. The Cambridge ARM model uses Hoare triple theorems and separation logic [19] to describe the behavior of each instruction, and the model captures realistic details of ARM instructions, which we illustrate briefly. The model decompiles each ARM instruction to a Hoare triple theorem of the form ( $p$ ) c ( $q$ ), where $p$ and $q$ are predicates describing the state of the processor before (pre-state) and after (poststate) executing code $c$ respectively ${ }^{1}$. Then, the theorem (p) c (q) informally means that for a processor in a state satisfying $\mathrm{p}$ before running $\mathrm{c}$, after running $\mathrm{c}$, the processor will have state satisfying $\mathrm{q}$. The predicates $\mathrm{p}$ and $\mathrm{q}$ are pre- and post-state assertions about the values of machine resources e.g., registers, status flags and the program counter. They can also contain pure boolean assertions which describe relationships among the values of machine resources which are true before or after an instruction executes. The theorem for the ARM instruction 0xE5832000 (mnemonic "str r2 [r3]") is:

$\vdash$ SPEC ARM MODEL $($ aR 3w $r 3 *$ aR 2w $r 2 *$ aPC $p *$ aMEMORY $d f f$

$* \operatorname{cond}((r 3 \& \& 3 w=0 w) \wedge(r 3 \in d f)))\{(p, 0 x E 5832000 w)\}$

$($ aR 3w $r 3 *$ aR 2w $r 2 *$ aPC $(p+4 w) *$ aMEMORY $d f((r 3=+r 2) f))$

SPEC indicates that the theorem is a Hoare triple, while ARM_MODEL is the ARMspecific instruction semantics [15]. aR 2w and aR 3w are expressions which assert that a given register stores the specified value, where $2 \mathrm{w}$ and $3 \mathrm{w}$ indicate the register number whose value is being asserted, and the suffix w indicates the register number is a fixed-width word. Then, the pre-state shows that the registers $\mathrm{r} 2$ and $r 3$ contain the (symbolic) values $r 2$ and $r 3$ respectively, the main memory contains the map $f$ with domain $d f$, and the program counter has some address $p$ before running the instruction. After running the instruction, the values of registers $r 2, r 3$ remain unchanged, and the program counter advances to $p+4$. Also, $=+$ is the map-update operator, hence $r 3=+r 2$ indicates that the memory has been updated to store the value that was in register $\mathrm{r} 2$ at the address given by the value that was in register $r 3$. The expression cond $((r 3 \& \& 3 w=0 w) \wedge(r 3 \in d f))$ is an assertion which specifies our memory alignment requirement for writes to the address $r 3$, and that $r 3$ is in the domain of the memory map $f . *$ is the separating conjunction [19] which asserts all other resources are unchanged.

\footnotetext{
${ }^{1}$ In Hoare logic, p, q are named pre-, post-condition, but we use the terms pre-,
} post-state as we call the boolean conditions imposed by a branch the pre-condition. 


\subsection{Composition rule in Hoare logic}

$\frac{S P E C x p c_{1} q \quad S P E C x q c_{2} r}{S P E C x p\left(c_{1} ; c_{2}\right) r}$ COMPOSE $\frac{S P E C x p c q}{S P E C x(p * r) c(q * r)}$ FRAME

The Compose rule of Hoare logic [11] is shown above, which extends single instruction Hoare triple theorems to describe multiple instructions. One critical detail of this rule is that to apply the Compose rule to compose two Hoare triple theorems, the pre-state of the second theorem must be equal to the post-state of the first theorem. Conceptually, when instruction $i_{1}$ executes, followed by instruction $i_{2}$, as $i_{2}$ is executing immediately after $i_{1}$, so the processor state just before $i_{2}$ executes is exactly the processor state after $i_{1}$ executes.

Pre-composition Tactic. A typical proof tactic for composing Hoare triple theorems for sequential instructions, $i_{1}, i_{2}$, with $i_{1}$ running immediately before $i_{2}$, into a single Hoare triple theorem, is given by the following steps: (i) Using the Frame rule (shown above), add machine state assertions in $i_{1}$, but not in $i_{2}$, to $i_{2}$ 's theorem; (ii) Using the Frame rule, add machine state assertions in $i_{2}$, but not in $i_{1}$, to $i_{1}$ 's theorem; (iii) Instantiate free variables in $i_{2}$ with the post-state machine resource values from $i_{1}$. We call these steps the pre-composition tactic. This is similar to the "shift" operation described by Myreen et al. [15]. After carrying out the above theorem manipulation steps, the manipulated theorems $i_{1}^{\prime}$ and $i_{2}^{\prime}$ for both instructions will now have the post-state of $i_{1}^{\prime}$ matching the pre-state of $i_{2}^{\prime}$, allowing us to directly apply the Compose rule in Hoare logic.

For instance, consider the two instructions, $i_{1}$ ("mov $\mathrm{r} 3, \mathrm{r} 4$ "), followed by $i_{2}$ ("sub r2, r3, \#16"). We illustrate the use of the Compose rule to obtain a theorem describing the behavior of a program (or its fragment), $i_{1} i_{2}$. The Hoare triple theorems for each of the two instructions are shown respectively:

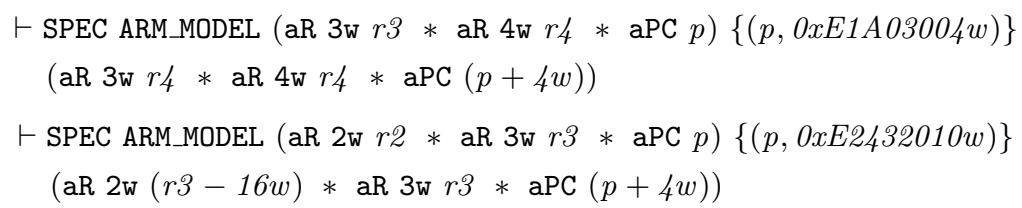

Thus, in composing the two theorems $i_{1}, i_{2}$ in our above example, our precomposition tactic will carry out the following steps on the theorems $i_{1}, i_{2}$ : (i) Use the Frame rule to add aR $2 \mathrm{w} r 2$ to $i_{1}$ to get $i_{1}^{\prime}$; (ii) Use the Frame rule to add aR $4 \mathrm{w} r 4$ to $i_{2}$ to get $i_{2}^{\prime}$; (iii) Instantiate the value of $p$ to $p+4 w$, and $r 3$ to $r 4$ in $i_{2}^{\prime}$ to get $i_{2}^{\prime \prime}$; (iv) Apply Compose rule to theorems $i_{1}^{\prime}, i_{2}^{\prime \prime}$ to obtain:

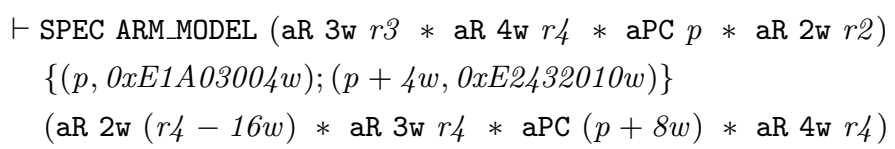

The pre-composition tactic prepares two suitable Hoare triples for reasoning about the effects of code on the same pre-state (i.e. pre-state of the first Hoare triple) by placing them in the same context (i.e. describing the effects of the code in both triples in terms of the pre-state variables of the first Hoare triple). 


\section{Design: The $\mathcal{L}_{L R}$ Program Logic}

Next, we describe the design of our logic framework for automatically verifying safety properties, and discuss the rationale behind our design decisions. Our logic framework needs to fulfill three tasks: (1) Specify safety assertions for each instruction. A safety assertion of an instruction specifies the conditions which must be true before the instruction is executed for our safety properties to hold. (2) Ensure that the Hoare triple theorems for every instruction are encoded with their safety assertions. (3) Define, formally, the requirements for a program to possess our desired safety properties.

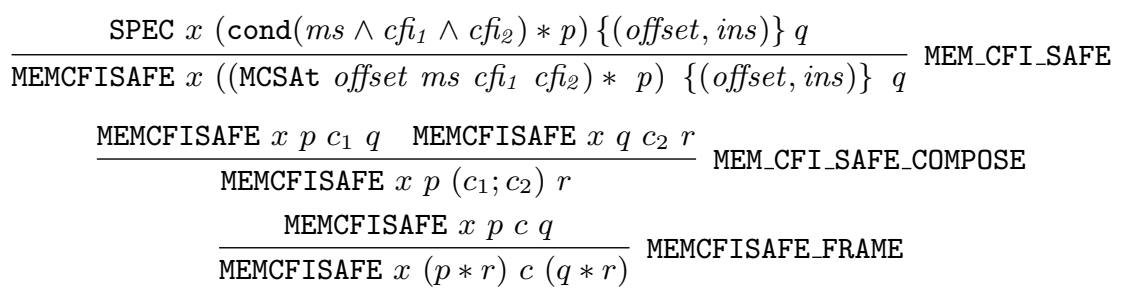

Fig. 2. Logic rules for $\mathcal{L}_{L R} . m s, c f_{1}, c f_{2}, c f_{3}$ are safety assertions for memory and control-flow isolation respectively. MCSAt is a syntactic label to group safety assertions.

\subsection{Individual Instructions: Safety Assertion Specification}

Figure 2 shows the MEM_CFI_SAFE rule for augmenting the Hoare triple theorem of a single instruction with its safety assertion. This rule overcomes the challenge of reasoning about safety properties at every instruction using Hoare logic. We add our safety assertions as a pure boolean condition to the pre-state of an instruction's Hoare triple. Then, when the Compose rule (\$2.4) is applied to compose theorems of multiple instructions, the pre-states of successor instructions ( $q$ in the Compose rule) will be hidden, thus hiding our augmented safety assertions. Also, safety assertions which hold can be simplified to true and eliminated from the Hoare triple. Thus, for a Hoare triple describing a sequence of instructions, we cannot tell if the theorem contains safety assertions for every instruction.

The MEM_CFI_SAFE rule overcomes this challenge by ensuring that the Hoare triple for every instruction has been augmented with its safety assertions. This rule has two features. First, MEM_CFI_SAFE can be instantiated only from single instruction Hoare SPEC theorems, because code $c$ in the SPEC theorem in the rule antecedent admits only a single instruction with the machine word ins located at address offset. Also, the second rule which generates safe MEMCFISAFE theorems, MEM_CFI_SAFE_COMPOSE, does not admit Hoare triple SPEC theorems, and only allows the composition of MEMCFISAFE theorems. Second, the MEM_CFI_SAFE rule can be instantiated only when the pre-state is augmented with our safety assertion, the pure boolean conjunction, $m s \wedge c f i_{1} \wedge c f i_{2}$, in its pre-state. Thus, the MEMCFISAFE relation indicates the resulting Hoare triple has been augmented with safety assertions for every instruction described. MCSAt is a syntactic relation which associates our safety assertion, $m s \wedge c f i_{1} \wedge c f i_{2}$, with the address 
offset which the assertion applies to. We also add the safety assertions $m s, c f i_{1}$, $c f i_{2}$ to the hypotheses of the theorem, to indicate that they are undischarged.

Safe instruction semantics are sound. Our safe instruction semantics, in the form of MEMCFISAFE theorems, are a special form of Hoare triple theorems. They are augmented to ensure that every instruction described in an MEMCFISAFE theorem has an associated safety assertion, added to it as a pure boolean condition in the pre-state of the instruction's theorem. We proved the following theorem: $\vdash \forall x p c q$. MEMCFISAFE $x p c q \Rightarrow$ SPEC $x p c q$. Informally, our safety-augmented Hoare triple theorems retain a direct correspondence to the Hoare triple theorems proven by the Cambridge ARM model. Hence, our safe instruction semantics inherits the soundness of the Cambridge ARM model.

\subsection{Sequential Code Blocks}

Next, we describe how we obtain safety-augmented Hoare triple theorems for basic blocks of sequential code (safe basic block theorems). A basic block is a sequence of instructions which execute sequentially, with a single entry and single exit instruction. The two rules (Fig. 2) we need for building safe basic block theorems are MEM_CFI_SAFE_COMPOSE, and MEMCFISAFE_FRAME (proved using the Frame rule in separation logic). These two rules allow us to inductively build up a safe basic block theorem from safety theorems for individual instructions. The process of building up a safety theorem for a basic block of sequential code is the same as that of composing Hoare triple theorems (§2.4), except that only safetyaugmented Hoare triple theorems can be composed. This process is repeated recursively for every instruction in a basic block to obtain a single safe theorem for the basic block. Our safe basic block theorems have the same semantics as Cambridge ARM Hoare triples, as proved in $\S 3.1$.

\subsection{Function Judgment for Local Reasoning}

Global vs. Local Reasoning. In a typical correctness proof for a program using Hoare logic, we would repeatedly apply the Compose rule to the Hoare triple for every instruction in the program to obtain a single Hoare triple describing the entire program. This is a "global reasoning" process which identifies the final values of all registers, main memory, etc. at the end of the program's execution. In the presence of loops and function calls, loop invariants and preand post-conditions for functions will need to be manually provided.

For safety assertions to hold in a program, we only need to ensure that the safety assertions for each instruction hold locally at that instruction. For the safety assertions at instruction $i_{2}$ to hold, we consider every instruction $i_{1}$ that can execute immediately before $i_{2}$. The machine-resource values in the poststate of each $i_{1}$ must satisfy the safety assertions at $i_{2}$. This is analogous to the pre-composition process $(\S 2.4)$. As long as the machine-resource values in the post-states of predecessor instructions $i_{1}$ enable the safety assertion at $i_{2}$ to be true, the safety assertion holds. Also, any pure boolean condition from the poststate of predecessor instructions $i_{1}$ will also apply to the pre-state of instruction 


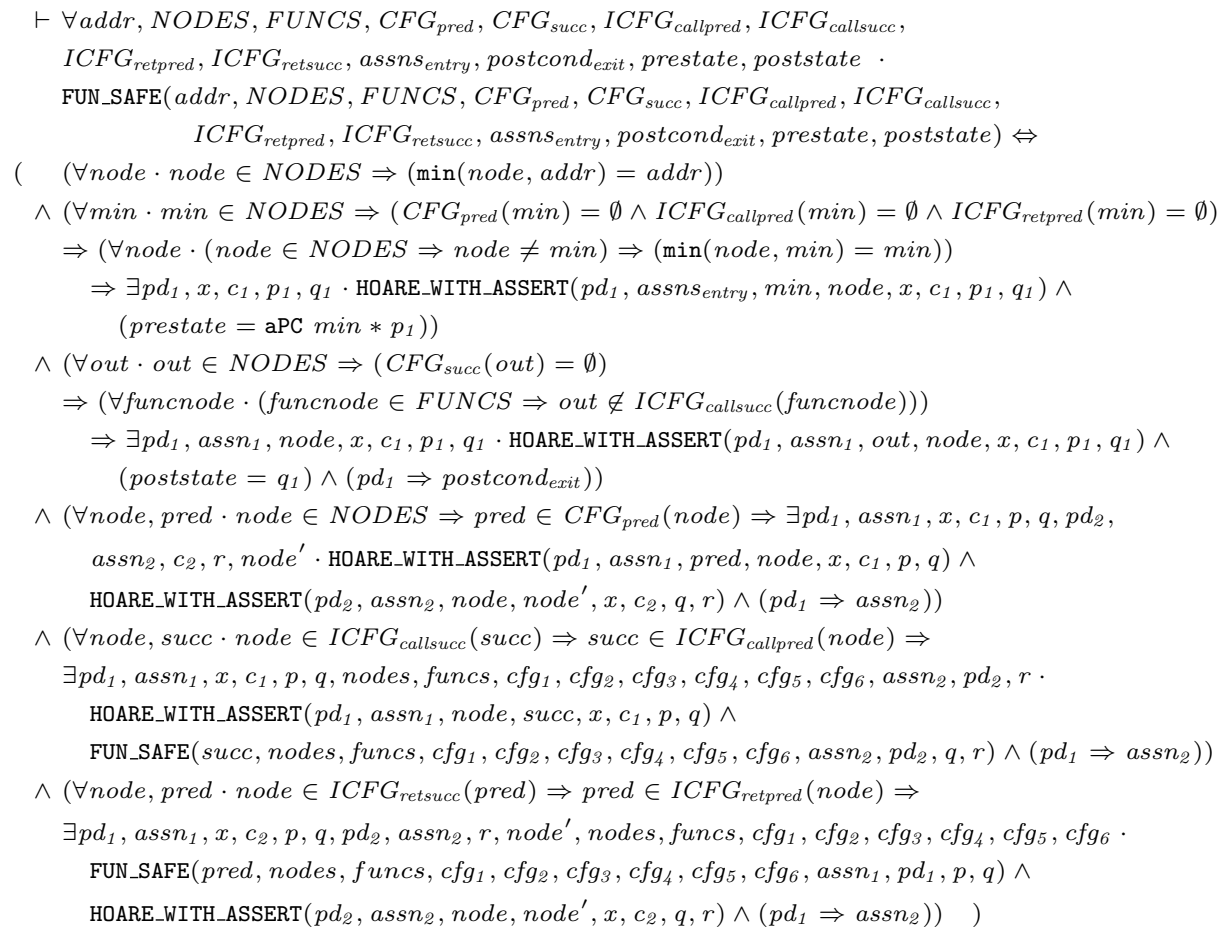

Fig. 3. FSI rule: Judgment for Interprocedural Function Safety

$i_{2}$. Hence, safety properties hold on a per-instruction basis. To check if a safety assertion holds for an instruction, we only need to perform "local reasoning" by considering the post-state and boolean conditions of all predecessor instructions. Safe Function Judgment. We define the FUN_SAFE rule (Fig. 3), which encodes what it means for a function to be safe. This rule encodes our "local reasoning" process for verifying that safety assertions hold. Thus, proving that the machine-code of a given function is safe involves proving that the FUN_SAFE theorem holds for the function. First, we rearrange MEMCFISAFE theorems to form HOARE_WITH_ASSERT theorems, which make explicit the hypotheses (i.e., undischarged safety assertions) of the theorems, and rearrange machine resource expressions into tuples for pattern-matching.

$$
\begin{aligned}
& \vdash \text { HOARE_WITH_ASSERT }\left(p d, a s s n, p c_{p r e}, p c_{p o s t}, x, c, p, q\right) \Leftrightarrow \\
& \quad\left(a s s n \Rightarrow\left(\operatorname{MEMCFISAFE~} x\left(\operatorname{aPC} p c_{p r e} * p * \text { precond } p d\right) c\left(\operatorname{aPC} p c_{p o s t} * q\right)\right)\right)
\end{aligned}
$$

A function is comprised of basic blocks of instructions in the function. In a function's intra-procedural control-flow graph (CFG), nodes are basic blocks of the function's instructions, while edges are control transfers within the function. In a function's inter-procedural CFG, the nodes are (i) basic blocks which call other functions, (ii) basic blocks which are return-sites from callee functions, and (iii) callee functions, while edges are function calls or returns. To formally specify the requirements for a function to be safe, we consider the safety assertions which 
must be discharged at each edge in both the intra- and inter-procedural CFGs. We walk through each of the 6 conjunct clauses in the FSI rule in Figure 3.

Arguments to the FUN_SAFE relation. The FUN_SAFE relation is parameterized by the function address $a d d r$, a set of addresses of basic blocks in the function NODES, a set of addresses of callee functions $F U N C S$, and 6 maps $C F G$ and $I C F G$ specifying the predecessors and successors of edges in the function's intra- and inter-procedural CFGs. FUN_SAFE also records, for a function, the safety assertions assns entry $_{\text {, the conditions which hold at its exit postcond }}$ exit, and the machine resource pre-state prestate and post-state poststate.

Function entry and exit specifications. The first clause states that the address of the function is the lowest basic block address for the function. The second clause states that the safety assertions assns $s_{\text {entry }}$ and pre-state prestate of the function are specified by the entry basic-block of the function. The third clause states that the function's guaranteed exit condition postcond $_{\text {exit }}$ and poststate poststate are specified by the exit basic-block of the function.

Intra-procedural safety requirements. The fourth clause specifies that for each intra-procedural CFG edge, the safety assertions of the instruction at the destination of each edge must be discharged by the post-condition of the instruction at the source of the edge, i.e., $\left(p d_{1} \Rightarrow a s s n_{2}\right)$. Also, in the spirit of the Hoare Compose rule, we require that the post-state of the predecessor instruction $q$, is equal to the pre-state of the successor instruction.

Inter-procedural safety requirements. The fifth and sixth clauses specify the requirements for inter-procedural CFG edges. The fifth clause specifies that for call edges, the safety assertions of the called function must be discharged by the post-condition of the calling basic block $\left(p d_{1} \Rightarrow a s s n_{2}\right)$. The sixth clause specifies that for return edges, the safety assertions of the basic block which is the return site for the function must be discharged by the post-condition of the returning function $\left(p d_{1} \Rightarrow a s s n_{2}\right)$. In both clauses, we require that the post-state of the predecessor node must equal the pre-state of the successor node.

Compositional reasoning for functions. Although the FSI rule appears to be recursively defined without a base case, this rule actually collapses to include only the first four clauses for functions which do not call any other functions. This implies that our safety property proving requires the CFG of the program to have no cycles, i.e. we are unable to analyze recursive programs.

\section{Implementation: Proofs using $\mathcal{L}_{L R}$}

We describe the implementation of our automatic safety property verification. Our framework consists of 128 lines of HOL 4 definitions and 11.8 KLOC of proof scripts in ML. Algorithm 1 summarizes the overall workflow of the AUSPICE safety property proof process. First, AUSPICE computes basic blocks and extracts function boundaries from the machine-code of the program (Line 14). Next, AUSPICE obtains the Hoare triple theorems from the Cambridge ARM model for each machine-code instruction (Line 15), adds safety assertions to the Hoare triple theorem for each instruction (§4.1), and composes the individual in- 
structions' theorems into a single Safe Basic Block theorem for each basic block (Line 16). AUSPICE's proof process takes place on a per-function basis beginning from the entry-function. For each function, all callee functions called by that function are analyzed before the function itself is analyzed (Line 3). Next, AUSPICE applies the Selective Composition tactic ( $\$ 4.2)$ to the safe basic block theorems to propagate branch conditions and function prologue information to the appropriate theorems for the function (Lines 6 and 7). The main process for discharging safety proof obligations is the SafetyAssertionAnalysis function (Line 8), which implements the proof search process using abstract interpretation (§4.3). Then, the results of the assertion analysis are applied to each of the basic blocks' theorems, and the FSI_rule function (Line 10) generates the FUN_SAFE safety theorem for the target function being proved to be safe.

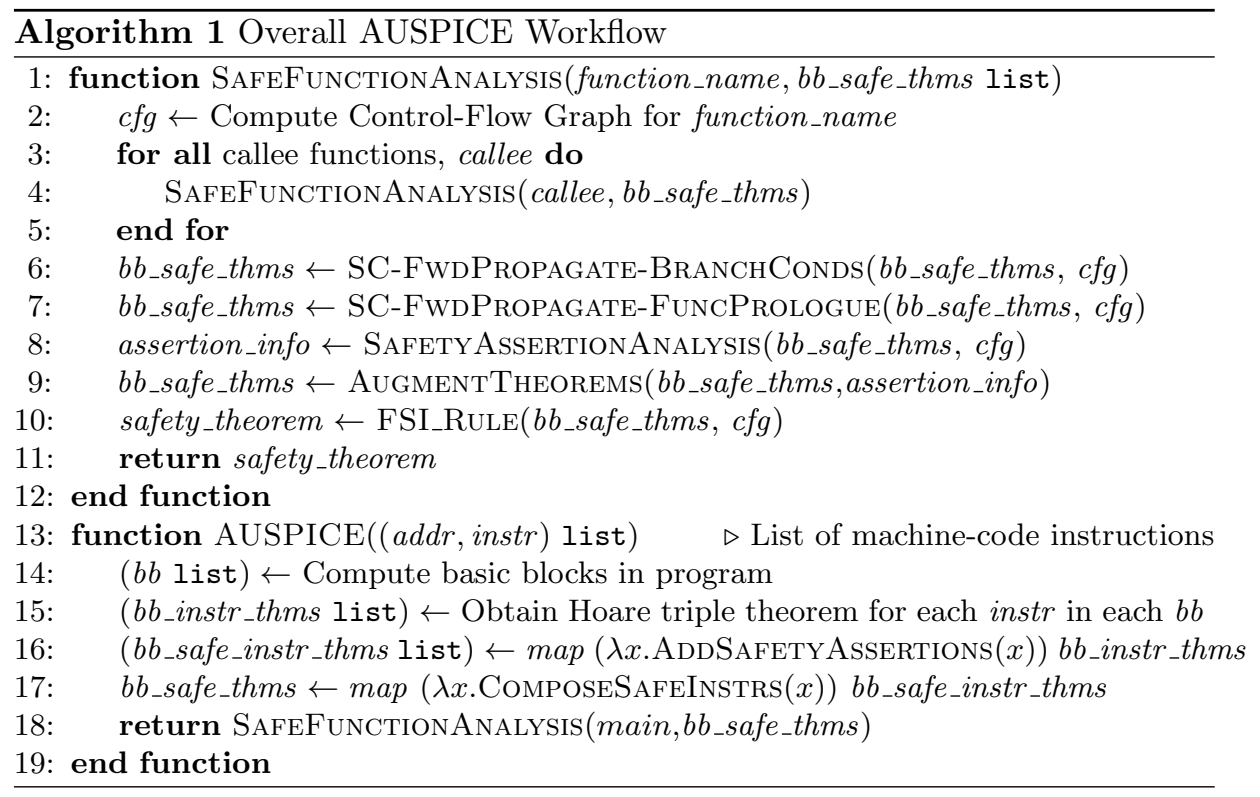

\subsection{Automatic Safety Property Specification}

To illustrate the safety assertions we augment instructions with, consider the instruction word 0xE5832000 (str r2 [r3]) located at address 0x81E0. We first obtain the following Hoare logic theorem from the decompiler:

$\vdash$ SPEC ARM_MODEL $(\mathrm{aR} 3 \mathrm{w} r 3 *$ aR $2 \mathrm{w}$ r2 $*$ aPC $(0 x 81 E 0) *$ aMEMORY $d f f$

$* \operatorname{cond}((r 3 \& \& 3 w=0 w) \wedge(r 3 \in d f)))\{(0 x 81 E 0,0 x E 5832000 w)\}$

$($ aR 3w $r 3 *$ aR 2w $r 2 *$ aPC $(0 x 81 E 4) *$ aMEMORY $d f((r 3=+r 2) f))$

Suppose the text section of this program lies in the range [0x80B4, $0 x 85 F 4]$. This instruction writes to the byte locations $r 3, r 3+1, r 3+2, r 3+3$. Thus, we 
set the first conjunct in the safety assertion $m s$ to $\{r 3+3 ; r 3+2 ; r 3+1 ; r 3\}$

$\subseteq\{a d d r \mid 0 \times 85 \mathrm{~F} 8 \leq a d d r \wedge a d d r \leq 0 \mathrm{xBF} 000000\}$ which asserts that the memory locations written to are in our allowed safe region. Then, the first control-flow safety conjunct, $c f i_{1}$ is set to $\exists p c . p c=0 \times 81 \mathrm{E} 4 \wedge p c \in$

$\{a d d r \mid$ 0x80B4 $\leq a d d r \wedge a d d r \leq 0 \times 85 F 4\}$, which asserts that the address of the next instruction to be executed lies in the text section of the binary. Next, the second control-flow safety conjunct, $c f i_{2}$ is set to $\{r 3+3 ; r 3+2 ; r 3+1 ; r 3\} \subseteq$ $\{a d d r \mid a d d r<\mathrm{r} 11\}$, which asserts that the memory locations written to cannot overwrite the saved link register ( $l r$, stored in register $r 11$ ) value on the stack.

\subsection{Selective Composition Proof Tactic}

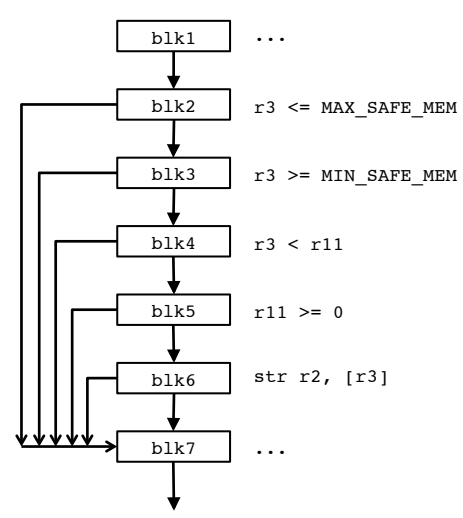

Fig. 4. Possible structure for program with safe str r2 [r3].
Next, we discuss the steps for automatically proving that safety properties hold using $\mathcal{L}_{L R}$. After augmenting single instruction theorems with safety assertions ( $\$ 3.1$ ) and obtaining safe basic block theorems ( $\S 3.2)$, we need to prove that the antecedents in the FSI rule (Fig. 3) hold. Each of the top-level conjuncts of FSI requires either a HOARE_WITH_ASSERT theorem for safe basic blocks or a FUN_SAFE theorem for safe functions. We also need to prove that the pre-condition $p d_{1}$ of each predecessor CFG node discharges the safety assertion $a_{s s n_{2}}$ in the successor CFG node.

From $\S 4.1$, we can see that the safety assertion at each instruction contains three conjuncts: one for memory-isolation and two for control-flow isolation. In a safe program, for the theorem of a given instruction $i_{2}$, its predecessor (safe basic block or function) theorem $i_{1}$ should have a pre-condition which implies the safety assertion of $i_{2}$. Observe that the safety assertion for each instruction has three conjuncts, and each of the range conjuncts ( $m s$ and $c f i_{1}$ in $\S 4.1$ ) is specified by two conjuncts: one each for the lower and upper bounds of the valid memory locations written to. Thus, the safety assertion at each instruction comprises multiple conjuncts. However, in a machine-code program, each basic block can only carry out one of the "elementary" arithmetic comparison operations (one of $<,>, \leq, \geq$, etc.), because each cmp* instruction is a branch and will mark the end of the basic block it belongs to. Hence, information from multiple predecessor basic blocks are required to discharge the safety assertion at each instruction.

Forward propagation of branch conditions. In $\S 3.3$, we noted that we must use a local reasoning process to ensure our proof process is automatic, because global reasoning would require manually-specified information. However, our safety assertions contain multiple conjuncts, whereas each basic block in machine-code can provide only one conjunct in its pre-condition. To enable our proof process to use pre-conditions from predecessors which are more than one 


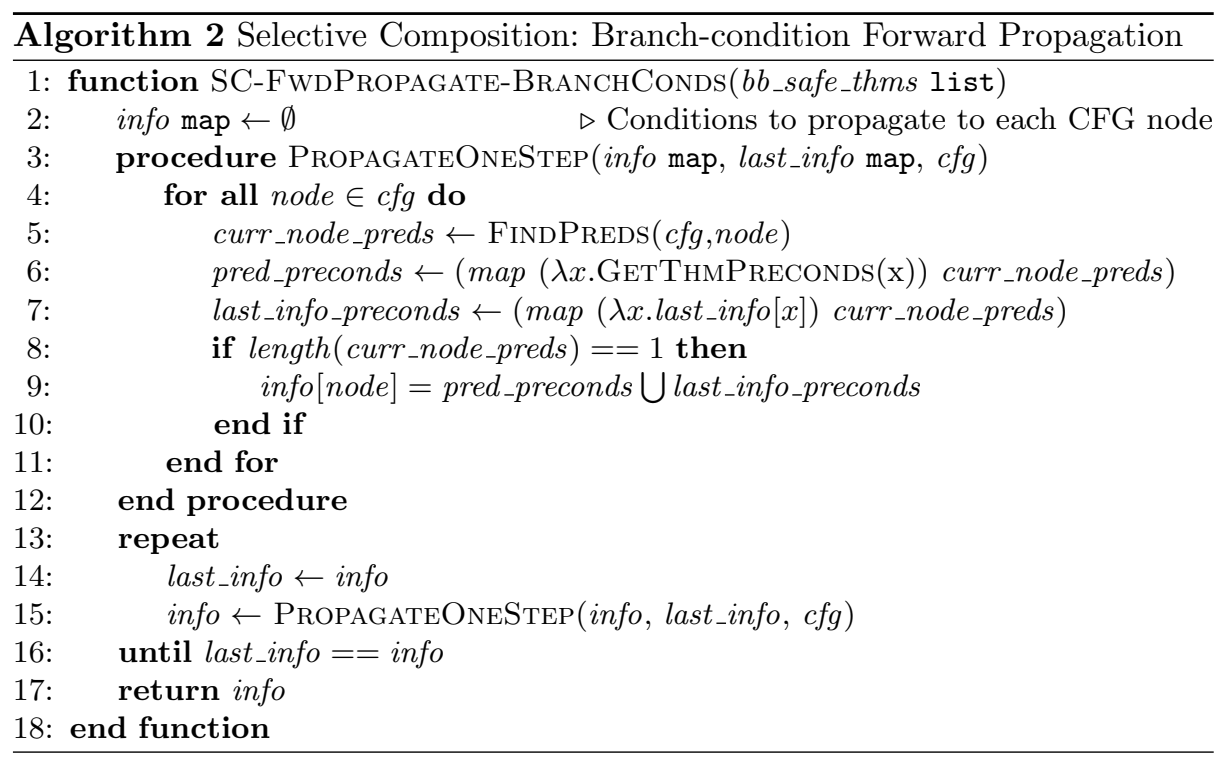

edge away from a given basic block in the program CFG, we selectively "propagate" the pre-conditions of basic blocks forward. We call this process "selective composition", where we apply the pre-composition tactic (§2.4) forward to successor theorems under certain conditions.

To illustrate the process of selective composition, consider, for example, the store instruction str r2 [r3]. Figure 4 shows the CFG of the possible structure of the basic blocks in a program with safety checks to ensure that the store instruction is safe. Then, we need the pre-conditions from basic blocks $b l k_{2}, b l k_{3}, b l k_{4}, b l k_{5}$ to be available at $b l k_{5}$ to discharge the safety assertion at $b l k_{6}$. At each of the nodes $b l k_{2}, b l k_{3}, b l k_{4}, b l k_{5}$, there are two Hoare triple theorems: one where each $b l k_{i}$ executes $b l k_{i+1}$ next (for $i \in\{2,3,4,5\}$ ), and one where the safety check fails, and each $b l k_{i}$ goes on to execute $b k_{7}$. However, we do not compose $b l k_{2}, b l k_{3}, b l k_{4}, b l k_{5}$ to form a single Hoare triple theorem, because the resulting block of code will have multiple exits, which is not captured by our safe basic block theorem (the MEM_CFI_SAFE_COMPOSE rule), which only admits single-exit blocks. Instead, we iteratively apply the pre-composition tactic (§2.4) for basic blocks $b l k_{2}, b l k_{3}, b l k_{4}, b l k_{5}$. This lets us place the analysis of the machine-code in blocks $b l k_{2}, b l k_{3}, b l k_{4}, b l k_{5}, b l k_{6}$ in the context of the prestate values of machine resources in blk . This then allows us to discharge the safety assertion at $b l k_{6}$ with the combined pre-conditions of $b l k_{2}, b l k_{3}, b l k_{4}, b l k_{5}$ at $b l k_{5}$. We call this process "selective composition" because we carry out the pre-composition process without applying the composition rule. Note that this selective composition process succeeds only when the target basic block which the pre-conditions are being propagated forward to have only one predecessor basic block. Only then is the pre-condition from the predecessor block $b l k_{i}$ the only pre-condition that will apply at the successor block $b l k_{i+1}$. 
Algorithm 2 describes the Selective Composition tactic for the forward propagation of branch-conditions in pseudocode. The tactic uses a fixed-point intraprocedural static-analysis over the Hoare triple theorems of a function. The static-analysis identifies branch-conditions to propagate forward from each theorem to its successor theorems (Lines 3 to 16; FindPreds returns the predecessors for a given node in the CFG of the function, while GetThmPreconds returns the pre-conditions for a given Hoare triple theorem). The analysis also ensures that branch-conditions are propagated forward only when the target node has only one predecessor in the CFG (Line 8). The analysis returns the branch-conditions to add to each node's theorem in the program's CFG (Line 17).

Local use of global information. Next, we describe the second instance of selective composition. Recall that for control-flow isolation, we require that the address of each instruction executed must be within the text section of the program. The address of the next instruction to be executed can be statically determined at every point of the program except where a function returns to its caller. Consider a typical machine-code instruction for returning from a function call pop $\{\mathrm{pc}\}$. Control is being returned from the function by restoring the saved link register value from the stack to the program counter. The instruction will be specified by the Hoare triple theorem:

$\vdash$ SPEC ARM_MODEL (aPC $p *$ aR 13w $r 13 *$ aMEMORY $d f f * \operatorname{cond}(((f r 13) \& \& 3 w=0 w) \wedge$

$$
((f r 13) \in d f)))\{(p, 0 x E 8 B D 8000 w)\}(\operatorname{aPC}(f r 13) * \text { aR 13w }(r 13+4 w) * \text { aMEMORY } d f f)
$$

Here, aMEMORY df $\mathrm{f}$ is an assertion that the main memory is the map $f$ which when applied to an address $a d d r$, returns the word stored at $a d d r$, and $d f$ is a set specifying the address domain of $f$. Thus, in the post-state of this instruction, we can see that the next instruction to be executed is at address $f$ r13. However, the memory map $f$ does not contain any information that enables us to determine the value of $f$ r13. The return address for a (non-leaf) function is saved to the stack in the function prologue before any instructions in the function. An example of such an instruction is push $\{l r\}$, with the following Hoare triple:

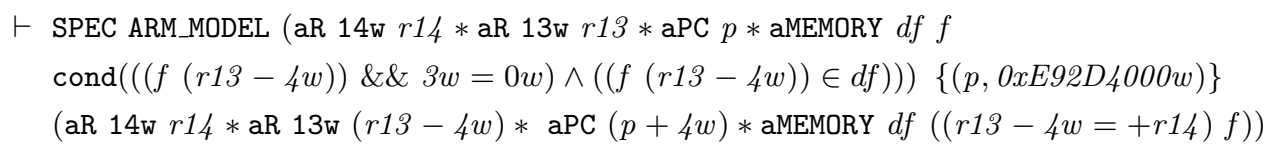

The memory in the post-state of the function is $((r 13-4 w=+r 14) f)$, which contains the value of the link register, $r 14$, at the top of the stack, at the address $r 13$ - 4. Hence, the information we need to discharge the control-flow safety assertion at the function exit is the memory expression at the post-state of the function prologue, and the new value of register $r 13$. After substituting the post-state memory and register $r 13$ values of the function prologue into the return instruction, the program counter in the return instruction post-state will contain $((r 13-4 w=+r 14) f) \quad(r 13-4 w)$ which simplifies to $r 14$, and the safety assertion simplifies to $r 14 \in\{$ addr $\mid 0 \times 85 F 8 \leq$ addr $\wedge$ addr $\leq 0 \times 85 F 4\}$, which can be discharged by any caller of the function, which supplies a concrete value of $\mathrm{r} 14$. As long as the prologue precedes every instruction in the function, and the function does not alter the callee-saved registers until its epilogue, this substitution is valid. Again, we can use the pre-composition tactic 
to substitute the value of the memory (and registers) at the post-state of the function prologue into every subsequent basic block in the function. Unlike the forward-propagation of branch-conditions, a fixed-point analysis is not required, and we directly substitute the information from the function prologue in every subsequent basic block in the function.

\subsection{Automatic Discharge of Proof Obligations}

There are two ways to discharge the safety assertions of a theorem. First, for a given safety theorem, the pure boolean conditions of the pre-state of the theorem preceding it may imply the safety assertion holds for the current theorem. Second, if the former does not hold, then the safety assertion is added to the hypotheses of the preceding instruction, and the Frame rule is used to add the undischarged assertion to the theorems of the preceding instructions. We use abstract interpretation [7] to identify safety assertions which cannot be discharged. At each instruction, our analysis records the safety assertions which need to be framed to the safe instruction theorem for that instruction.

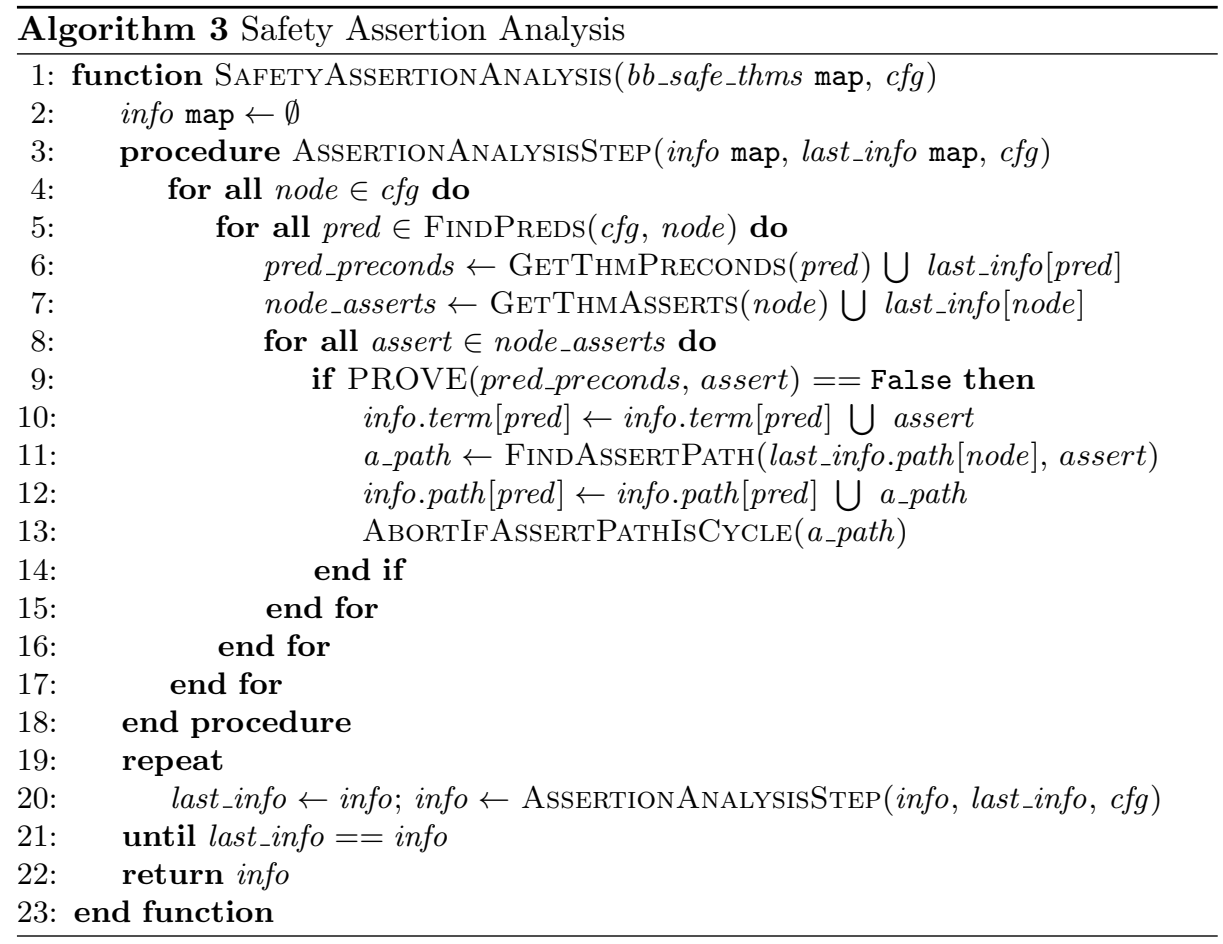

We use a flow-sensitive backwards fixed-point analysis. Our analysis proceeds across all nodes in the CFG of a function in reverse topological order in each iteration. Each CFG node is a basic block in the function, and each node is 
associated with a safe basic-block theorem (§3.2). At each node, the analysis checks that for each predecessor node, the instruction theorem for that node has pure boolean conditions which can discharge the safety assertions at the current node's theorem. For safety assertions which the predecessor node's theorem cannot discharge, our analysis adds the assertion to the predecessor node's theorem, propagating the assertion backwards up the CFG. Our analysis is also inter-procedural, and context-sensitive. Each function is summarized at its callsite by a FUN_SAFE theorem for that particular call-site.

In the general case, this analysis may not terminate. If there are safety assertions being propagated which have values that change with a loop, the analysis will not terminate: the free variable instantiation at loop boundaries will generate new safety assertions to be framed whenever the assertion is propagated across the back-edge of the loop. We prevent the assertion analysis from running forever by (i) recording the propagation path of safety assertions, and (ii) aborting the analysis if a cycle is detected on this path. Then, we inform the user that we are unable to prove our safety properties for the program.

Algorithm 3 describes our static-analysis algorithm. GetThmPreconds (Line 6) and GetThmAsserts (Line 7) return the pure boolean conditions in the prestate and the safety assertion at a node's theorem respectively. PROVE tries to discharge the given safety assertion, assert, using the given conditions pred_preconds from the predecessor theorem, and returns true if it can discharge the safety assertion, and false otherwise (Line 9). If the safety assertion cannot be discharged, it is added to the analysis information for the node's predecessor node (Line 10), so that it will be framed to the predecessor node's theorem after the analysis. The analysis information also records the path along which each assertion is propagated in info.path (Line 12). Then, the analysis checks if there is a cycle along the propagation path of the assertion (Line 13) in the function AbortIfAssertPathIsCycle, and terminates the AUSPICE proof process if a cycle is found. This is because if a cycle is found along which the pre-composition tactic causes the safety assertion term to change with each iteration, the analysis is likely to not terminate as it will keep adding new safety assertion terms to the analysis information on each successive iteration of the analysis.

\section{Discussion}

Soundness of Proof Rules. AUSPICE's proof rules for single instruction (§3.1) and basic block (§3.2) safety are sound, because we derive our MEM_CFI_SAFE, MEM_CFI_SAFE_COMPOSE, and MEMCFISAFE_FRAME proof rules from the Hoare triples for machine-code programs in the Cambridge model, which Myreen et al. have shown to be sound [16]. Also, using the HOL4 proof assistant to define our proof rules further ensures they are sound. In addition, we proved (§3.1) that safe single instruction and basic block theorems in AUSPICE derived from our proof rules have the same instruction semantics as the ARM machine-code semantics defined by the trustworthy, validated Cambridge ARM model $[9,15]$. 
Correctness of Safety Rule. Next, we give a brief, informal argument of the correctness of our proof rule for safe programs. The FUN_SAFE theorem (Figure 3) can be proven for a program if and only if safety assertions are specified for every instruction, and if these safety assertions hold before that instruction begins executing (except for the first instruction, which relies on the OS to correctly initialize the processor state for the program). We argue this by Structural Induction on the Control-Flow Graph (CFG) of a program. Each node in our CFG of a function in a program is either a single-entry, single-exit basic block with sequentially executing code, or a (callee) function called by the function.

Base Case. The MEM_CFI_SAFE rule (§3.1) ensures every instruction's theorem contains our safety assertions ( $\$ 2.2)$. The MEM_CFI_SAFE_COMPOSE rule ensures every basic block's theorem is built up only from single-instruction theorems with added safety assertions. The requirement that post-states of predecessor theorems and pre-states of successor theorems must be equal in MEM_CFI_SAFE_COMPOSE ensures every basic block's theorem accumulates the safety assertions for every composed safe instruction theorem. Then, for a program with only a single instruction or basic-block, if the OS correctly initializes the processor state, the safety assertions will hold for the single instruction or single basic block.

Inductive Case. We take the CFG of a function, $G$, and partition its vertices into a single vertex, $g$, and all other vertices, $G^{\prime}$. By the Inductive Hypothesis, the FUN_SAFE theorem holds for $G^{\prime}$. Then, consider the edges $E$ connecting $G^{\prime}$ to $g$. In the absence of function pointers and unstructured jumps (longjmp), the edges $E$ are either (i) intra-procedural control-flow transfers between basic blocks in the function, (ii) function calls from a basic block in the function to a callee function, or (iii) function returns from a callee function to a basic block in the function. Then, for FUN_SAFE to be true, the fourth to sixth conjunct clauses of the FUN_SAFE rule must be true, so the pre-conditions of the theorems of all predecessor vertices to $g$ in the CFG discharge the safety assertions at $g$, making the safety assertions at $g$ hold, for any type of possible control-flow transfer to $g$. Thus, our FUN_SAFE rule ensures that we have captured all the possible control-flow transfers in a machine-code program. For FUN_SAFE to be correct, we require correct $\mathrm{CFG}$ predecessor and successor maps, which are straightforward to compute without function pointers and unstructured jumps.

Limitations. Our machine-code safety properties $(\S 2.2)$ have been formulated to ensure they can be automatically proven to hold in machine-code programs. These properties are sufficient, but not strictly necessary to meet our highlevel goal of preventing the control-flow of a machine-code program from being hijacked due to user input. The strictness of our safety properties helps automate the verification process. Our requirement that machine-code instructions do not alter memory addresses greater than the current function's frame pointer address also prevents functions from modifying any variables passed by reference from the stacks of caller functions. Instead, functions passing data by reference must store this data either on the program's heap, or use memory allocated in the program's data section. We believe this is a small inconvenience to enable the fully automated verification of safety properties. 


\section{Evaluation}

We aim to show that we can verify real-world programs, and we pick programs with constructs which are challenging to verify. We also measure our runtime to show the feasibility of our verification. Our test programs were compiled using an unmodified gcc toolchain for the ARMv7 architecture with -00 optimization. Figure 5 summarizes our test programs. sort implements Bubble Sort, which has a doubly-nested loop, and also contains 2 other functions to exercise our inter-procedural analysis. memcpy is an implementation of the the $\mathrm{C}$ library function which we developed, and shows we can verify a real-world function. stringsearch is an application in the MiBench commercially-representative embedded benchmark [10], and it implements the Boyer-Moore string search algorithm, and demonstrates our verification on real-world programs.

\begin{tabular}{|c|c|c|l|}
\hline Test Program & Instructions & Functions & Description \\
\hline memcpy & 116 & 2 & Real-world memcpy \\
\hline sort & 337 & 5 & Nested loops, function calls/returns \\
\hline stringsearch & 530 & 5 & Boyer-Moore string search (MiBench [10]) \\
\hline
\end{tabular}

Fig. 5. Test programs, their sizes, and the purpose of each test.

\begin{tabular}{|c|l|l|l|l|l|}
\hline & $\begin{array}{l}\text { Cambridge ARM } \\
\text { Decompiler }\end{array}$ & $\begin{array}{l}\text { Safe Basic } \\
\text { Blocks }\end{array}$ & $\begin{array}{l}\text { Abstract In- } \\
\text { terpretation }\end{array}$ & $\begin{array}{l}\text { Safe Func- } \\
\text { tion }\end{array}$ & $\begin{array}{l}\text { Total Proof } \\
\text { Time }\end{array}$ \\
\hline memcpy & $1.3 \mathrm{mins}$ & $2.7 \mathrm{mins}$ & $5.7 \mathrm{mins}$ & $6.7 \mathrm{mins}$ & $16.4 \mathrm{mins}$ \\
\hline sort & $2.5 \mathrm{mins}$ & $11.2 \mathrm{mins}$ & $36 \mathrm{mins}$ & $73 \mathrm{mins}$ & $122.7 \mathrm{mins}$ \\
\hline stringsearch & $2.8 \mathrm{mins}$ & $15.3 \mathrm{mins}$ & $327.6 \mathrm{mins}$ & $17.8 \mathrm{mins}$ & $363.5 \mathrm{mins}$ \\
\hline
\end{tabular}

Fig. 6. Verification runtime.

Figure 6 shows the time taken to verify the safety of each of our test programs. We carried out the verification on an $2.6 \mathrm{GHz}$ Core i7 system. The majority of the verification time is spent in the abstract interpretation (\$4.3) and the proof of the safe function theorem ( $\S 3.3)$. We believe these are feasible times for verifying safety properties, as programs only need to be verified once on installation.

\section{Related Work}

Many techniques have been developed for verifying machine-code programs using logic. Certified assembly programming uses a Hoare logic with separation logic to build certified libraries $[26,18]$, but specifications must be manually annotated in programs, and verification is interactive. Tan and Appel [21] developed a program logic for multi-entry, multi-exit machine-code fragments to reason about unstructured control-flows in executables in Foundational Proof Carrying Code (FPCC). They require a special compiler to generate machine-code annotated with types [13], while we verify unmodified executables compiled using an offthe-shelf compiler. iTalX [22] infers types for x86 assembly programs, reducing the amount of type annotations required from a modified compiler. Executables have also been verified without using a program logic, although concise 
theorems cannot be proven. Bedrock [6] provides "mostly-automated" verification for generic program properties, and provides memory safety as a side-effect, for programs written using its idealized machine language, from which concrete architectures can be targeted. Xu et al. [25] verify safety properties for machinecode using static-analysis. RevGen [5] decompiles machine-code to the intermediate representation of the LLVM compiler framework, enabling other analyses to be reused, whereas we use a validated model of ARM machine-code. Thakur et al. [23] perform model-checking on machine-code without requiring a precomputed, fixed, inter-procedural CFG. Sequoll [4] also performs model-checking on machine-code programs, and like our work, uses the Cambridge ARM model [15], but it uses temporal logic to reason about worst-case execution time (WCET) in the NuSMV model-checker, whereas our approach uses Hoare logic. XFI [8] and ARMor [27], are software fault isolation (SFI) [24] implementations which ensure and verify that (x86 and ARM, respectively) executables possess memory and control-flow safety properties. XFI requires modules being verified to be annotated with hints. PittSFIeld [12] verifies that its SFI safety rewriting for $\mathrm{x} 86$ binaries is correct, as opposed to verifying that the executables it produces are safe. RockSalt [14] also provides verified SFI by providing a verified checker which checks that programs are isolated, whereas our work produces a safety proof for each program. ARMor [27] is closest to our work. They require machine-code to be compiled with a modified compiler, after which the program must undergo binary rewriting to insert safety checks. In contrast, we can prove safety properties automatically for unmodified executables using our logic framework and selective composition proof tactic.

\section{Conclusion and Future Work}

We have presented a novel logic framework, AUSPICE, for automatically verifying safety properties in unmodified ARM machine-code programs. Our framework consists of a program logic, $\mathcal{L}_{L R}$, which uses a subset of a trustworthy Hoare logic for ARM executables [15, 16], and extends it for local reasoning, and the selective composition proof tactic, which fully automates the verification of safety properties. We demonstrated the feasibility of our fully automated safety property verification on one synthetic and two real-world (including a real-world benchmark [10]) examples. In future, we intend to validate our approach on more programs, and expand our verification to programs with system calls.

\section{Acknowledgment.}

We thank Lu Zhao for his help with ARMor [27], Magnus Myreen for his help with the Cambridge ARM model $[15,16]$, and Xinyu Zhuang for his feedback.

\section{References}

1. The ARM-THUMB Procedure Call Standard (2000), http://infocenter.arm.com/help/topic/com.arm.doc.espc0002/ATPCS.pdf 
2. ARM Architecture Reference Manual, ARMv7-A and ARMv7-R edition (2014)

3. Abadi, M., Budiu, M., Erlingsson, U., Ligatti, J.: Control-flow Integrity. In: ACM CCS (2005)

4. Blackham, B., Heiser, G.: Sequel: A Framework for Model Checking Binaries. In: IEEE RTAS (2013)

5. Chipounov, V., Candea, G.: Enabling Sophisticated Analyses of x86 Binaries with RevGen. In: HotDep (2011)

6. Chlipala, A.: Mostly-Automated Verification of Low-Level Programs in Computational Separation Logic. In: PLDI (2011)

7. Cousot, P., Cousot, R.: Abstract Interpretation: A Unified Lattice Model for Static Analysis of Programs by Construction or Approximation of Fixpoints. In: POPL (1977)

8. Erlingsson, U., Abadi, M., Vrable, M., Budiu, M., Necula, G.: XFI: Software Guards for System Address Spaces. In: OSDI (2006)

9. Fox, A.: Formal specification and verification of ARM6. In: TPHOLs (2003)

10. Guthaus, M.R., Ringenberg, J.S., Ernst, D., Austin, T.M., Mudge, T., Brown, R.B.: Mibench: A free, commercially representative embedded benchmark suite. In: IEEE WWC Workshop (2001)

11. Hoare, C.A.R.: An axiomatic basis for computer programming. Commun. ACM 12(10) (Oct 1969)

12. McCamant, S., Morrisett, G.: Evaluating SFI for a CISC Architecture. In: USENIX Security (2006)

13. Morrisett, G., Crary, K., Glew, N., Grossman, D., Samuels, R., Smith, F., Walker, D., Weirich, S., Zdancewic, S.: TALx86: A Realistic Typed Assembly Language. In: Workshop on Compiler Support for System Software (WCSSS) (1999)

14. Morrisett, G., Tan, G., Tassarotti, J., Tristan, J., Gan, E.: RockSalt: Better, faster, stronger SFI for the x86. In: PLDI (2012)

15. Myreen, M., Fox, A., Gordon, M.: Hoare Logic for ARM Machine Code. In: Fundamentals of Software Engineering (FSEN) (2007)

16. Myreen, M., Gordon, M.: Hoare Logic for Realistically Modeled Machine Code. In: TACAS (2007)

17. Myreen, M., Gordon, M., Slind, K.: Machine-code verification for multiple architectures: An application of decompilation into logic. In: FMCAD (2008)

18. Ni, Z., Shao, Z.: Certified Assembly Programming with Embedded Code Pointers. In: POPL (2006)

19. Reynolds, J.: Separation Logic: A Logic for Shared Mutable Data Structures. In: IEEE LICS (2002)

20. Slind, K., Norrish, M.: A Brief Overview of HOL4. In: TPHOLs (2008)

21. Tan, G., Appel, A.: A Compositional Logic for Control Flow. In: VMCAI (2006)

22. Tate, R., Chen, J., Hawblitzel, C.: Inferable Object-Oriented Typed Assembly Language. In: PLDI (2010)

23. Thakur, A., Lim, J., Lal, A., Burton, A., Driscoll, E., Elder, M., Andersen, T., Reps, T.: Directed proof generation for machine code. In: CAV (2010)

24. Wahbe, R., Lucco, S., Anderson, T., Graham, S.: Efficient Software-Based Fault Isolation. In: SOSP (1993)

25. Xu, Z., Miller, B., Reps, T.: Safety Checking of Machine Code. In: PLDI (2000)

26. Yu, D., Hamid, N., Shao, Z.: Building Certified Libraries for PCC: Dynamic Storage Allocation. In: ESOP (2003)

27. Zhao, L., Li, G., Sutter, B.D., Regehr, J.: ARMor: Fully Verified Software Fault Isolation. In: EMSOFT (2011) 